URL

\section{DNA sequencing and analysis}

454 Life Sciences

Applied Biosystems

Beckman Coulter

CLC bio

Cogenics

DNASTAR

GE Healthcare

Geospiza

Helicos Biosciences

Illumina

LI-COR Biosciences

MACROGENUSA

Pacific Biosciences

Promega

RainDance Technologies

Roche Applied Sciences

Saturn Biotech

Sequetech

SeqWright

SoftGenetics
Automated DNA analysis systems including Genome Sequencer 20 and FLX

Automated sequencing systems using both Sanger methodology and next-generation SOLiDbased system

Automated DNA sequencing systems based on capillary electrophoresis using the Sanger method

Developing software platforms for the analysis of next-generation sequencing data sets sequencing systems

Software for DNA sequence and microarray analysis

Kits for automated DNA sequencing systems

Software for management genetic data

Developing HeliScope Genetic Analyzer based on single-molecule sequencing methodology

Illumina Genome Analyzer next-generation DNA sequencing system

Automated DNA sequencing instrumentation using the Sanger methodology

DNA sequencing services

Automated next-generation sequencing instrument

fmol DNA sequencing system for manual DNA sequencing and promoter mapping applications

Microfluidic-based sequence-enrichment technologies

454 Life Sciences Genome Sequencer 20 and FLX systems

Full DNA sequencing service provider

DNA sequencing services

Contract automated DNA sequencing services

Software analysis tools for genetic research, including for ChIP sequencing and microRNA discovery
Contract automated DNA sequencing services using both Sanger-based and next-generation

\begin{tabular}{ll} 
Branford, Connecticut & www.454.com \\
Foster City, California & www.appliedbiosystems.com \\
Fullerton, California & www.beckmancoulter.com \\
Aarhus, Denmark & www.clcbio.com \\
Morrisville, North Carolina & www.cogenics.com \\
Madison, Wisconsin & www.dnastar.com \\
Buckinghamshire, UK & www.gehealthcare.com \\
Seattle, Washington & www.geospiza.com \\
Cambridge, Massachusetts & www.helicosbio.com \\
San Diego, California & www.illumina.com \\
Lincoln, Nebraska & www.licor.com \\
\hline Rockville, Maryland & www.macrogenusa.com \\
Menlo Park, California & www.pacificbiosciences.com \\
Madison, Wisconsin & www.promega.com \\
Lexington, Massachusetts & www.raindancetechnologies.com \\
Basel, Switzerland & www.roche.com \\
Perth, Australia & www.saturnbiotech.com.au \\
Mountain View, California & www.sequetech.com \\
Houston, Texas & www.seqwright.com \\
State College, Pennsylvania & www.softgenetics.com \\
\hline
\end{tabular}

\section{DNA microarrays}

Affymetrix

Agilent Technologies

Expression Analysis

Jivan Biologics

Marligen Biosciences

NanoString Technologies

NimbleGen

Oxford Gene Technology

PerkinElmer

Phalanx Biotech Group
Promoter, ENCODE, chromosome arrays; whole-genome tiling arrays

CpG islands, promoter, ENCODE arrays; custom tiling arrays; DNA methylation microarray software

Expression profiling and genotyping services

Microarrays and PCR for alternative splicing analysis

Transcription factor analysis, SNP genotyping, DNA and RNA purification kits

$\mathrm{n}$-Counter digital gene-expression analysis system using barcodes

Microarrays, software and services

Oligonucleotide arrays

Microarray scanners; genomics-analysis software

Whole-genome DNA microarray; gene-expression profiling service
Santa Clara, California

Santa Clara, California

Durham, North Carolina

Larkspur, California

ljamsville, Maryland

Seattle, Washington

Madison, Wisconsin

Oxford, UK

Waltham, Massachusetts

Hsinchu, Taiwan www.affymetrix.com

www.agilent.com

www.expressionanalysis.com

www.jivanbio.com

www.marligen.com

www.nanostring.com

www.nimblegen.com

www.ogt.co.uk

www.perkinelmer.com

www.phalanxbiotech.com

\section{Genomic services}

Epigenomics

Biomarker discovery services; kits for methylation analysis of biomarker genes; real-time PCR assays

Geneservice

Genomic and proteomic resources; contract services for DNA sequencing, microarray analysis and SNP genotyping

Genpathway

Whole-genome ChIP sequencing services; ChIP-on-chip assays

Berlin, Germany

Cambridge, UK

www.epigenomics.com

San Diego, California

www.geneservice.co.uk

Miltenyi Biotec

Microarray-based genomic services

Bergisch Gladbach, Germany

www.genpathway.com

www.miltenyibiotec.com

Operon Biotechnologies

Huntsville, Alabama

www.operon.com 


\begin{tabular}{|c|c|c|c|}
\hline COMPANY & PRODUCTS/ACTIVITY & LOCATION & URL \\
\hline QIAGEN & DNA, RNA extraction kits; genotyping; haplotyping & Hilden, Germany & www.qiagen.com \\
\hline Sequenom & $\begin{array}{l}\text { Genomic analysis services including genotyping, gene expression and methylation analysis, and } \\
\text { molecular typing }\end{array}$ & San Diego, California & www.sequenom.com \\
\hline SwitchGear Genomics & Promoter and UTR libraries; functional microarrays; DNA methylation analysis service & Menlo Park, California & www.switchgeargenomics.com \\
\hline TeleChem International & Microarray robots, printers and scanners; microarray services & Sunnyvale, California & www.arrayit.com \\
\hline Vermillion & Biomarker identification for drug discovery & Fremont, California & www.vermillion.com \\
\hline
\end{tabular}

\section{PCR}

Abgene

PCR and quantitative PCR reagents

Epsom, UK

www.abgene.com

Antarus Biotech

PCR instruments

Huntsville, Alabama

Clontech

Variety of molecular biology reagents including PCR products, gene-expression profiling and RNA Mountain View, California interference assays

Corbett Life Science

Real-time PCR cycler system; other life-sciences instruments

Sydney, Australia

Finnzymes

PCR reagents and thermal cyclers

Espoo, Finland

SABiosciences

Quantitative real-time PCR assays for ChIP analyses; microRNA arrays; protein phosphorylation assays

Sigma-Aldrich

Custom oligonucleotides; DNA sequencing systems; RNA interference reagents

Frederick, Maryland

St Louis, Missouri

Quantitative PCR reagents and instruments; microarray reagents; RNA and DNA purification kits

La Jolla, California

Oligonucleotides for PCR, real-time PCR assay development; PCR tests

Berlin, Germany

Bisulphite-treated DNA amplification; EZ DNA methylation kit

Orange, California

www.antarus.net

www.clontech.com

www.corbettlifescience.com

www.finnzymes.com

www.sabiosciences.com

www.sigmaaldrich.com

www.stratagene.com

www.tib-molbiol.com

www.zymoresearch.com

Zymo Research

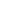

\section{General}

ALEXIS Biochemicals

Attagene

Cambrex

Cole-Parmer

EMD (Novagen)

EPICENTRE Biotechnologies

Evrogen

Fisher Scientific

Hamilton

Inivtrogen

Lonza

Maxim Biotech

Merck

MP Biomedicals

New England Biolabs

Takara Bio

USB

see advertisement
Reagents for molecular- and cell-biology research

Transcription-factor profiling system; software

Molecular- and cell-biology reagents and tools

Instruments and reagents

Reagents and kits for molecular biology

Enzymes for PCR and RT-PCR; DNA and RNA purification and isolation kits

Enrichment kits for transcriptomic applications; fluorescent-probe development

Research equipment, chemicals and kits

Robotics for the life sciences

Kits and reagents for molecular-biology, genomics and cell-biology research

Molecular-biology reagents and systems

PCR reagents and systems; nucleic-acid isolation; custom services including primer design, sample preparation and library construction

Chemicals, kits and reagents for molecular and cell biology-related research

Reagents and chemicals for research

Molecular biology-related reagents, kits and enzymes

Reagents, kits and services for genomics and molecular biology research

Chemicals and reagents for molecular biology
Lausanne, Switzerland

Research Triangle Park, North

Carolina

Charles City, lowa

Vernon Hills, Illinois

Madison, Wisconsin

Madison, Wisconsin

Moscow, Russia

Waltham, Massachusetts

Reno, Nevada

Carlsbad, California

Basel, Switzerland

South San Francisco, California

Darmstadt, Germany

Santa Ana, California

Ipswich, Massachusetts

Shiga, Japan

Cleveland, Ohio www.alexis-corp.com

www.attagene.com

www.cambrex.com

www.coleparmer.com

www.emdbiosciences.com

www.epibio.com

www.evrogen.com

www.fishersci.com

www.hamiltoncomp.com

www.invitrogen.com

www.lonza.com

www.maximbio.com

www.merck.de

www.mpbio.com

www.neb.com

www.takara-bio.com

www.usbweb.com

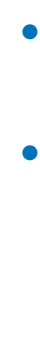

